\title{
Design of Adaptive Bit Interleaved TCM for Rayleigh Fading Channels
}

\author{
Vincent K.N. Lau \\ knlau@eee.hku.hk \\ Department of Electrical and Electronic Engineering \\ University of Hong Kong
}

August 26, 1999

\begin{abstract}
In this paper, we propose a bandwidth efficient error correction scheme, namely the variable rate adaptive bitinterleaved trellis coded modulation (ABICM), for wireless mobile channel. The code rate and modulation level are varied according to the current channel state to exploit the time-varying nature of the wireless channel. Design challenges to achieve symbol-by-symbol adaptation, component codes design, puncturing and interleaving design, adaptation thresholds determination are addressed. A multi-level puncturing scheme is proposed to tackle the problem of symbol-by-symbol adaptive puncturing and interleaving. We introduced the concept of equivalent distance spectrum for designing component codes of the ABICM system. Two operation modes, namely the constant throughput and the constant BER controls, are introduced. The design is illustrated with an example and it is found that there are significant gains relative to the fixed rate coding in terms of SNR and throughput. It is also found that the ABICM scheme is essentially not degraded in small interleaving depths. This makes the ABICM very suitable for real time applications.
\end{abstract}

\section{Introduction}

One important bottleneck in wireless communications is to find bandwidth-efficient modulation and channel coding schemes to protect data over hostile mobile radio channels. Trellis coded modulation, which is based on the idea of combining channel modulation and coding, was purposed[1] to offer high bandwidth-efficient protection. In [2], it has been shown that performance of TCM depends on the minimum number of distinct symbols ${ }^{1}$ between two codewords. To increase the code diversity, bit-interleaved coded modulation (BICM)[3], which breaks a modulation symbol with $b i t-b y$-bit interleaving, has been purposed. On the other hand, to better exploit the time varying nature of the channel, variable rate adaptive channel coding and modulation has been proposed $[4,5,6]$. The code rate and modulation level are varied according to the instantaneous channel condition. The receiver estimates the channel states and informs the transmitter through the use of a low capacity feedback link. Significant performance gains relative to

\footnotetext{
${ }^{1}$ Minimum number of distinct symbols between any two codewords is called the minimum Hamming distance.
}

fixed rate coding in terms of SNR and average throughput was obtained. In fact, the concept of variable rate adaptive coding and modulation has been widely applied in 3rd generation TDMA systems like Enhanced Data rate for GSM Evolution (EDGE) and UWC136.

It is therefore a natural extension to combine the above two concepts, namely the BICM and the variable rate adaptive channel coding, together to achieve a synergy. One very simple way to combine the two concepts is to adopt a multiframe adaptation technology similar to the EDGE system where the coding mode for a number of frames are fixed. To further enhance the performance gains, we have to adopt a symbol-by-symbol adaptation design. However, this is not a trival extension. Challenges yet to be solved include puncturing and interleaving design, component codes design and determination of optimal adaptation thresholds. In this paper, we address the above challenges. In section 2, we outline the system design of the proposed ABICM and propose a new multi-level puncturing and interleaving scheme that could solve the dilemma between interleaving and symbolby-symbol puncturing. Optimal adaptation thresholds are determined. In section 3, we discuss the code design issues. In section 4 , we illustrate the design with an example and study the performance gains for two operation modes, namely the constant BER and the em constant throughput. In particular, we show that the symbol-by-symbol ABICM scheme proposed is very robust to bursty channel errors and this is very useful when large interleaving depth is not feasible for some real time applications. Finally, we conclude with a brief summary of results in section 5 .

\section{Adaptive Bit-Interleaved Coded Modulation}

\subsection{System Design}

The simplified system block diagram of the proposed scheme is shown in fig. 4. The transmitter consists of a variable rate punctured convolutional encoder, a bit-by-bit interleaver, a variable throughput adaptive modulator, and a channel state predictor. The variable rate convolutional encoder instantaneouly varies the encoding rate by puncturing which periodically deletes coded bit streams based on some puncturing patterns ${ }^{2}$. A number of constellation

\footnotetext{
${ }^{2}$ In the design, incremental punctured code is used.
} 
modes are used at the modulator to provide variable modulation throughputs. When properly matched with the variable rate punctured codes, a number of modes with varying overall throughputs is available.

Channel states are estimated at the receiver and fed back to the transmitter via a low capacity feedback link with certain delay. Appropriate encoding modes and modulation constellations are determined symbol-by-symbol at the transmitter based on the fed-back channel states. At the receiver, given the transmission mode sequence, a single Viterbi decoder could be used to determine the ML received sequence for all modes.

\subsection{Multi-level Puncturing and Interleav- ing}

Interleaving is used to convert the bursty fading into in dependent fading. Puncturing is used to achieve variable rate encoding. However, for variable rate adaptive coding system with symbol-by-symbol adaptation, puncturing and interleaving presents a design problem.

For puncturing, the family of punctured codes have different puncturing periods which usually span over several symbol durations. In symbol-by-symbol adaptation, a number of mode changes within a period is possible and this destroy the rate-compatibility (or incremental puncturing) property of the punctured codes. Furthermore, when interleaving is considered, the puncturing and the interleaving tasks create potential conflicts. Conventionally, puncturing is followed by interleaving and followed by modulation. When coded bits enters the interleaver, the puncturing level for the bits has to be determined. However, the puncturing level is determined by the channel state when the bits are actually transmitted. There is always a huge interleaving delay from the time bits enter the interleaver and the time the bits are actually taken out of the interleaver and transmitted. This is a deadlock and is illustrated in figure 1. Therefore, puncturing could not be done before interleaving. On the other hand, puncturing could neither be done after interleaving. Otherwise, the designed puncturing pattern on the trellis diagram could not be maintained because it is possible that bits out of the interleaver actually correspond to non-puncturable bit positions in the original trellis diagram. This is illustrated in figure 2.

The solution to the problems is by multilevel puncturing and interleaving. We label the coded bits that are puncturable and defer the puncturing action after interleaving. Instead of a single block interleaver, we have a number of parallel interleavers of different levels. This is illustrated in figure 3 and figure 4 . Level- 0 interleaver contains coded bits that are non-puncturable. Level-1 interleaver contains coded bits that are puncturable according to puncturing pattern-1. Bits from level-0 interleaver must be transmitted by modulation symbols while bits from the other levels of interleavers are puncturable and therefore, could be transmitted or not transmitted (punctured).

With this layered approach, puncturing could be done after interleavers. Current channel state is obtained for every symbol duration, $T_{s}$. Based on the channel state, modula- tion symbol is determined and appropriate number of coded bits and puncturable bits are taken from appropriate levels of interleavers. This is illustrated in figure 3. For example, if 8 PSK is chosen, 3 coded bits are taken from the level0 interleaver and one puncturable bits are taken from the level-1 interleaver. The 3 coded bits are used to modulate a 8PSK symbol while the puncturable bit is not transmitted.

\subsection{Optimal Adaptation Thresholds}

Channel states are estimated at the receiver and fed back to the transmitter via a feedback link. Transmission mode $m$ is chosen if the predicted channel state, $\hat{z}$, falls between $\left[\zeta_{m}, \zeta_{m+1}\right] .\left\{\zeta_{m}\right\}$ is the adaptation thresholds which define how the ABICM operate as follows.

We have two different ways to operate the ABICM scheme, namely constant BER operation and constant throughput operation[5].

Constant BER Operation: The adaptation thresholds are set to maintain a relatively constant BER over a range of average SNR. Average throughput of the ABICM is compared relative to the fixed rate code at a given BER and at the same SNR.

Constant Throughput Operation: The adaptation thresholds are set to maintain a constant average throughput relative to the average SNR. SNR of the $A B I C M$ is compared relative to the fixed rate codes at a given BER and at the same throughput.

For the constant throughput operation, the optimal adaptation thresholds are $\left\{\zeta_{i}\right\}$ that minimize the BER with the constraint of constant average throughput. On the other hand, for the constant BER operation, the optimal thresholds are $\left\{\zeta_{m}\right\}$ that maximize the average throughput with the constraint of constant BER with respect to the SNR. It is shown in [7] that the optimal thresholds for both operation modes have the same form given by:

$$
\begin{aligned}
& {\left[\beta\left(\zeta_{1}, 1\right)-\beta\left(\zeta_{1}, 0\right)\right]=\left[\beta\left(\zeta_{2}, 2\right)-\beta\left(\zeta_{2}, 1\right)\right]} \\
& \quad=\cdots=\left[\beta\left(\zeta_{M-1}, M-1\right)-\beta\left(\zeta_{M-1}, M-2\right)\right]
\end{aligned}
$$

\section{Equivalent Distance Spectrum and Code Design}

The error performance of component code- $m$ is determined by its distance spectrum, $w_{m}(d, i)$, where $d$ is error path Hamming distance and $i$ is the information bits distance. For symbol-by-symbol variable rate adaptive codes, the resulting trellis is time varying and in general, the resulting distance spectrum depends on the specific mode sequence which in turn depends on the channel state, $\vec{\rho}$. As a consequence, the criteria to select the puncturing patterns of the component codes are not clear because there is no guarantee on the resulting distance spectrum even if we choose the best individual component codes. This difficulty is removed as a result of the multi-level puncturing arrangement. 
It is shown in [7] that the error performance of the $\mathrm{ABICM}$ is given by:

$$
P_{b}(\vec{\rho}) \leq \frac{1}{N_{c}} \sum_{d=1}^{\infty} \sum_{i=1}^{\infty} \tilde{w}(d, i) P(\vec{c} \rightarrow \overrightarrow{\hat{c}} \mid \vec{\rho})
$$

where $\bar{w}(d, i)$ is the equivalent distance spectrum. Therefore, the performance of symbol-by-symbol ABICM systems could be determined from its equivalent distance spectrum. It is also shown that $\bar{w}(d, i)$ is always bounded above and below by two adjacient modes, $w_{m}(d, i)$ and $w_{m+1}(d, i)$, for some $m$. Hence, the error performance of the ABICM code is bounded by the two nearest-neighbour fixed rate component codes and the criteria of code design is to select the best fixed-rate component codes with incremental puncturing pattern.

\section{Results and Discussion}

The ABICM design is illustrated with an example. A core rate- $1 / 2$ code with constraint length $K=5$ is used to generate a family of punctured codes with rates given by $[1 / 2,2 / 3,3 / 4,4 / 5,5 / 6]$. The family of constellations for the modulator is given by [BPSK, QPSK, 8PSK, 16QAM, 32QAM, 64QAM]. The available combined overall throughputs are listed below.

Mode-0: rate-1/2 +2 BPSK: throughput $=1 / 2$

Mode-1: rate-1/2 + 1 QPSK: throughput $=1$

Mode-2: rate-2/3 + 1 8PSK: throughput $=2$

Mode-3: rate-3/4 +1 16QAM: throughput $=3$

Mode-4: rate-4/5 + 1 32QAM: throughput $=4$

Mode-5: rate-5 $/ 6+1$ 64QAM: throughput $=5$

Note that throughput is defined as the number of information bits carried per modulation symbol. We assumed the forward baud rate is $500 \mathrm{k}$ baud with the symbol duration $T_{s}=2 \mu \mathrm{s}$. The maximum mobile speed of interest is $100 \mathrm{~km} / \mathrm{hr}$ and the corresponding Doppler frequency $f_{d} \approx 200 \mathrm{~Hz}$ at $2 \mathrm{GHz}$ band. The required feedback channel capacity is $7.2 \mathrm{kbps}$ which is much lower than the forward capacity.

The ABICM system is operated in constant throughput and constant BER operations. The performance in terms of $S N R$ gain and throughput gain is compared with the corresponding BICM schemes. Note that the SNR gains or the throughput gains are two sides of the same coin. It depends on the adaptation control to realize either one or both types of gains simultaneously.

\subsection{Ideal Performance of ABICM}

Constant Throughput Operation: The BER performance of the ABICM system relative to the fixedrate BICM system is plotted in figure 5. Note that along the BER curves of the ABICM scheme, the average throughput remains constant. Therefore, the relative SNR gains could be compared with the corresponding fixed rate BICM codes at the same throughput. For example, there are $4 \mathrm{~dB}, 5 \mathrm{~dB}, 5 \mathrm{~dB}$ SNR gains of the ABICM schemes relative to the 8PSK$\operatorname{BICM}(\bar{\gamma}=2), 16 \mathrm{QAM}-\mathrm{BICM}(\hat{\gamma}=3)$, 32QAM-BICM $(\vec{\gamma}=4)$ schemes respectively at BER level of $10^{-4}$.

Constant BER Operation: Figure 6 and 7 show the BER curves of the ABICM schemes operating in the constant BER mode. The BER levels off over a range of SNRs called the adaptation range. Outside the range, the SNR is either too large or too small and the ABICM is essentially operating on mode- 6 or mode- 0 respectively. Note that along the BER curves of the $\mathrm{ABICM}$ schemes, the average throughput is varying as illustrated in figure 7. To compare the performance with fixed-rate BICM schemes, we have to look at the throughput gains at the same BER and SNR points. For example, there are throughput gains of around 1.5 times at BER $=10^{-4}, 10^{-5}$ and $10^{-6}$ respectively.

\subsection{Effects of Block Interleaving}

Figure 8 illustrates the BER curves (at constant average throughput) of ABICM and BICM schemes with various interleaving depths at $f_{d} T_{s}=6 \times 10^{-3}$. Note that fixedrate BICM schemes are degraded significantly relative to the ideal interleaving situation at all throughputs. On the other hand, the ABICM schemes are much more robust to bursty channel errors. For example, the SNR gain from figure 8 becomes $10 \mathrm{~dB}$ relative to the 8PSK-BICM $(\bar{\gamma}=2)$ at $\mathrm{BER}=10^{-4}$ and interleaving depth of $64 \times 64$. Therefore, symbol-by-symbol adaptive channel coding schemes are good choices for protecting data over bursty channels when largedepth interleaving is not feasible for realtime applications.

\subsection{Effect of Feedback Delay and Noisy Feedback}

Estimated channel states are fed back to the transmitter with certain delay of $\Delta$ symbol durations. Assume the symbols on the feedback channel are transmitted with SNR $E_{f b} / \eta_{0}$. Figure 9 and 10 shows the degradations of ABICM with respect to $\Delta$ and $E_{f b} / \eta_{0}$. Since there is no interleaving in the feedback codewords, the required $E_{f b} / \eta_{0}$ for reasonable performance is quite large (about $20 \mathrm{~dB}$ ). However, since the baud rate on the feedback channel is much smaller than the forward channel ( 70 times or $18 \mathrm{~dB}$ ), the actual required transmitted power for the feedback codewords is not high at all.

\section{Conclusions}

In this paper, we propose a variable rate symbol-by-symbol adaptive error correction scheme error correction scheme for high bandwidth-efficiency applications. Design issues such 
as the symbol-by-symbol puncturing and interleaving, criteria for the choice of component codes, the formulation of optimal adaptation thresholds, the effect of feedback delay, noisy feedback and finite interleaving depth are addressed. For the first time, we introduce an optimal threshold control and multi-level puncturing to achieve symbol-by-symbol adaptation. We also show that the criteria of code design in ABICM is to choose (individually) the optimal fixed-rate component codes. It is found that there are significant SNR gains (up to $5 \mathrm{~dB}$ ) and throughput gains (up to 1.5 times) of the ABICM scheme. The scheme is also shown to be robust to bursty channel errors and feedback delay. Therefore, the scheme is very useful especially in cases where large interleaving depth is not be feasible.

\section{References}

[1] G. Ungerboeck, "Channel Coding with multilevel/phase signals," IEEE Trans. on Info. Theory, vol. IT-28, pp. 55-67, Jan. 1982.

[2] D. Divsalar and M. K. Simon, "Trellis Coded Modulation for $4800-9600 \mathrm{bits} / \mathrm{s}$ Transmission Over a Fading Mobile Satellite Channel," IEEE JSAC., vol. SAC-5, pp. 162-175, Feb. 1987.

[3] X. Li and J. A. Ritcey, "Trellis Coded Modulation with Bit Interleaving and Iterative Decoding," IEEE JSAC., vol. 17, pp. 715-724, A pril 1999.

[4] D. Cygan and E. Lutz, "A concatenated two-stage adaptive (CTSA) error control scheme for data transmission in time-varying channels," IEEE Trans. on Communs., vol. 43, pp. 795-803, Feb-March-April 1995.

[5] K. N. Lau and M. D. Malcolm, "Variable Rate Adaptive Trellis Coded QAM for High Bandwidth Efficiency Applications in Rayleigh Fading Channel," Special Issues on Mobile Comm. HKIE Transactions., August 1998.

[6] K. N. Lau, "Channel Capacity and Error Exponent of Variable Rate Adaptive Channel Coding for Rayleigh Fading Channel," To appear in IEEE Trans. on Communs.

[7] K. N. Lau, "On the design of Variable Rate, Symbol-bySymbol, Adaptive Bit Interleaved Trellis Coded Modulation.," Submitted to IEEE JSAC., 1999.

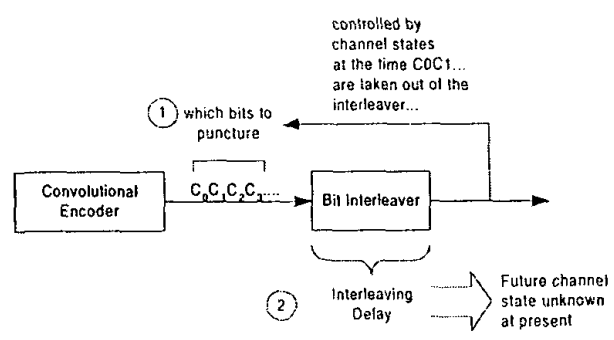

Figure 1: Conflict of Adaptive Puncturing before interleaving.

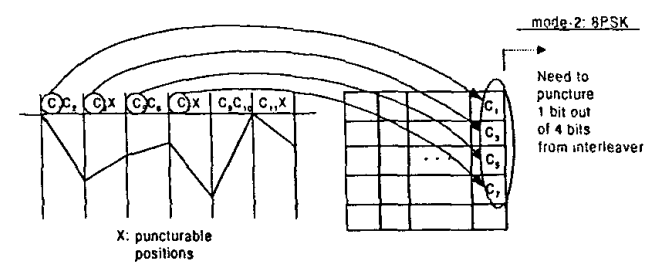

Figure 2: Conflict of Adaptive Puncturing after interleaving.

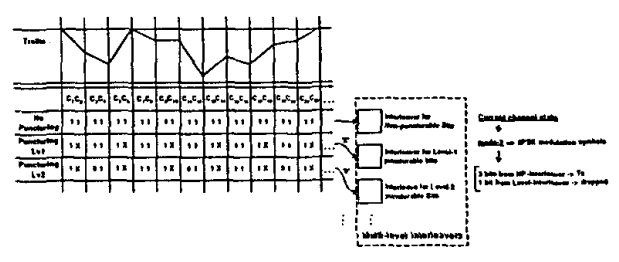

(a)

Figure 3: Multi-level Puncturing:

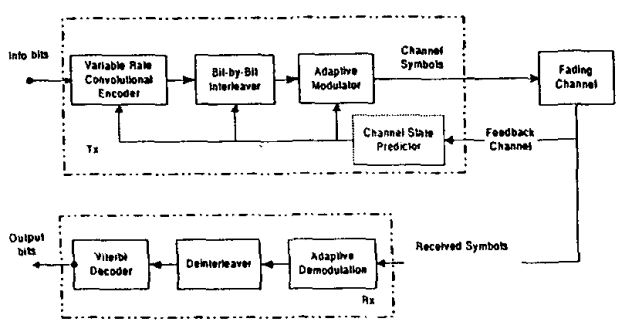

Figure 4: System Block Diagram. 


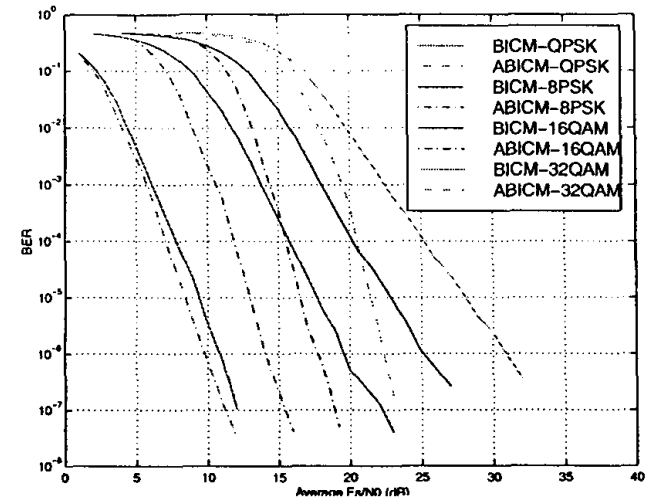

Figure 5: Performance of ABICM at constant throughput control.

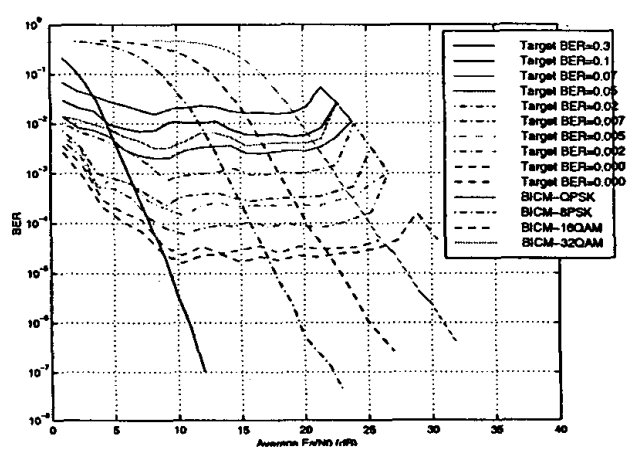

Figure 6: BER against SNR at constant BER control

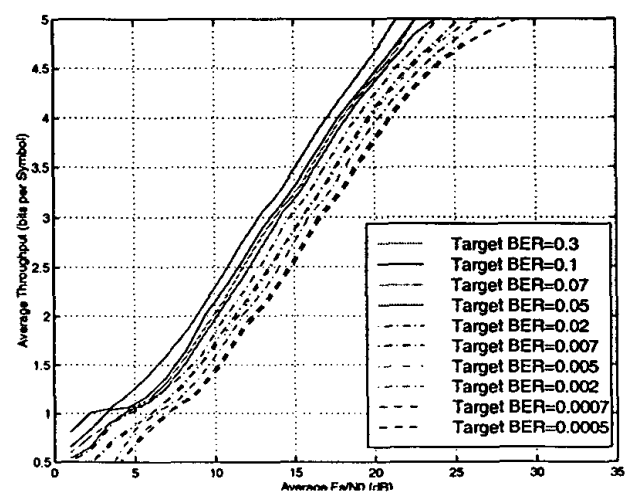

Figure 7: Throughput against SNR at constant BER control.

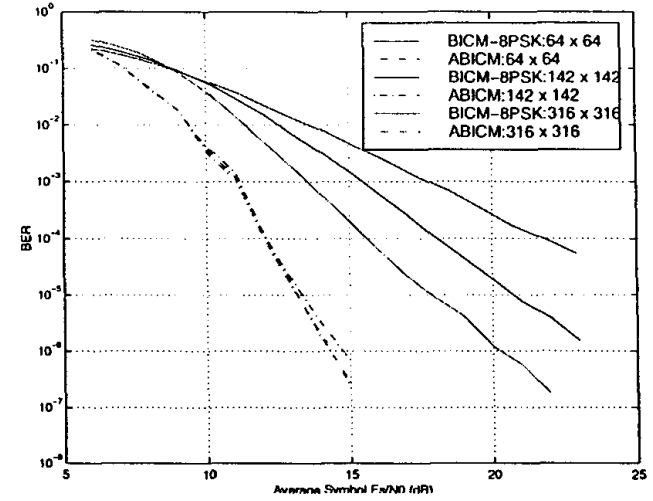

Figure 8: BER against SNR for ABICM-8PSK

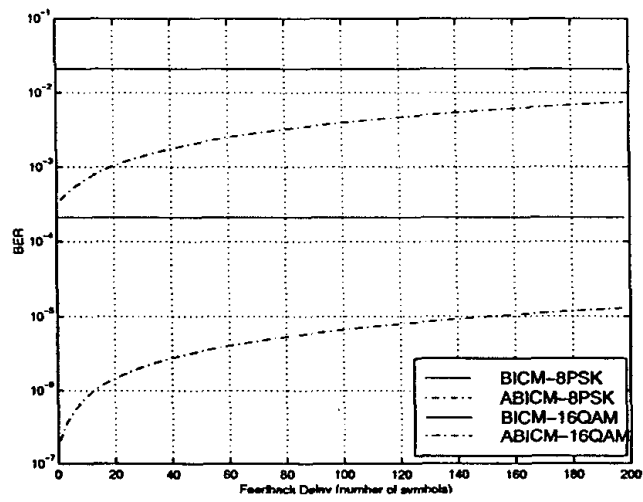

(a)

Figure 9: Effect of Feedback Delay

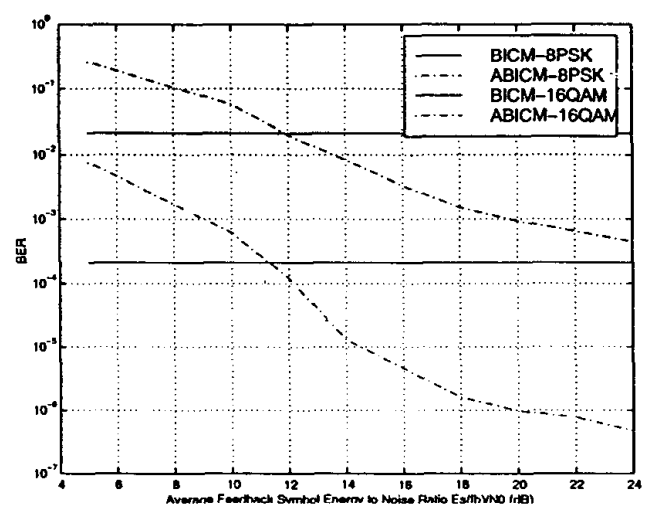

Figure 10: Effect of Feedback Noise. 